\title{
Structural Study of a Carbon Nanofiber Silicon Composite Electrode for Advanced Lithium Batteries
}

\author{
M. P. Balogh *, M. Nazri ** and G. A. Nazri* \\ * General Motors Company, GM Technical Center, Warren, MI 48090-9055 \\ ** 48090Applied Science Incorporate, GM Technical Center, Warren, MI 48090-9055
}

Lithium ion batteries with high specific and volumetric capacity are candidates for many applications including large format power source for transportation [1]. Currently, lithium intercalated carbonaceous anodes are used in most commercial lithium cells [2]. The energy density of the carbonaceous materials is limited to $372 \mathrm{mAh} / \mathrm{g}$ and may not satisfy the energy demand of future technologies. In addition, due to the low voltage of lithiated graphite, close to the deposition potential of metallic lithium, there is the potential for deposition of metallic lithium and thus formation of internal shorting in the cell during high charge acceptance of the cell. Therefore the next generation of anodes for advance lithium batteries will most probably be a lithium alloy.

Lithium alloys are especially interesting due to their high gravimetric and volumetric energy density. Among various lithium alloys, the lithium silicon has the highest energy density among all known alternative anodes. Theoretical specific capacity of lithium-silicon alloy is $4008 \mathrm{mAh} / \mathrm{g}$ with volumetric energy density of $9339 \mathrm{Ah} / 1$, corresponding to $\mathrm{Li}_{21} \mathrm{Si}_{5}$, over one order of magnitude higher than the energy capacity of the current lithium-carbon anode [3]. Although greater energy density of the lithium alloys has been recognized before, its application in real lithium batteries has not been realized. One of the major issues with lithium alloys particularly for Li-Si alloy is the large volume change which occurs during cycling. The large volume change during charge-discharge causes great stress in the silicon lattice and subsequently cracks and pulverizes the anode material. The high reactivity of lithiated silicon toward electrolyte decomposition and huge enhancement of its surface area causes cracking and delamination of the silicon anode from the current collectors and loss of electronic contact that leads to marked capacity loss during charge-discharge cycles [4].

In recent years attention has focused on the use of amorphous silicon that lacks phase changes during the lithiation-delithiation process. Amorphous silicon also has a more open structure and therefore less susceptible to crack formation during charge-discharge cycling [5]. Various silicon containing composite matrix, particularly with carbon and graphitic particulates, have been proposed to mitigate the problem of electronic conductivity loss during volume changes. However, limited success has been achieved to solve the poor cyclability of the Li-Si anode. During the last few years, we have developed a unique composite of carbon nanofiber silicon - composite (Si-CNF) anode and tuned the energy capacity of the composite for an advance lithium battery. The Si-CNF composite consist of an amorphous film deposited on carbon nanofibers as demonstrated in Figure 1. During cycling, the amorphous silicon does not show any sign of pulverization but rather forms a unique nano-sheet structure oriented perpendicular to the carbon fiber axis with an open structure. This unique structure has provided reversible lithiation delithiation process, forming a high energy capacity electrode for a lithium battery.

\section{References}

[1] J. M. Tarascon, M. Armand, Nature 404 (2001) 359. 
[2] G.-A. Nazri, G. Pistoia, Lithium Batteries Science and Technology, Kluwer Academic Publisher, 2004, p.113.

[3] J. Li, J. Dahn, J. Electrochem. Soc. 154 (2007) A156.

[4] S. Mukerjee and S. Srinivasan, Journal of Electroanalytical Chemistry, 357 (1993) 201.

[5] D. Larcher et al., J. Materials Chemistry 17 (2007) 3759.
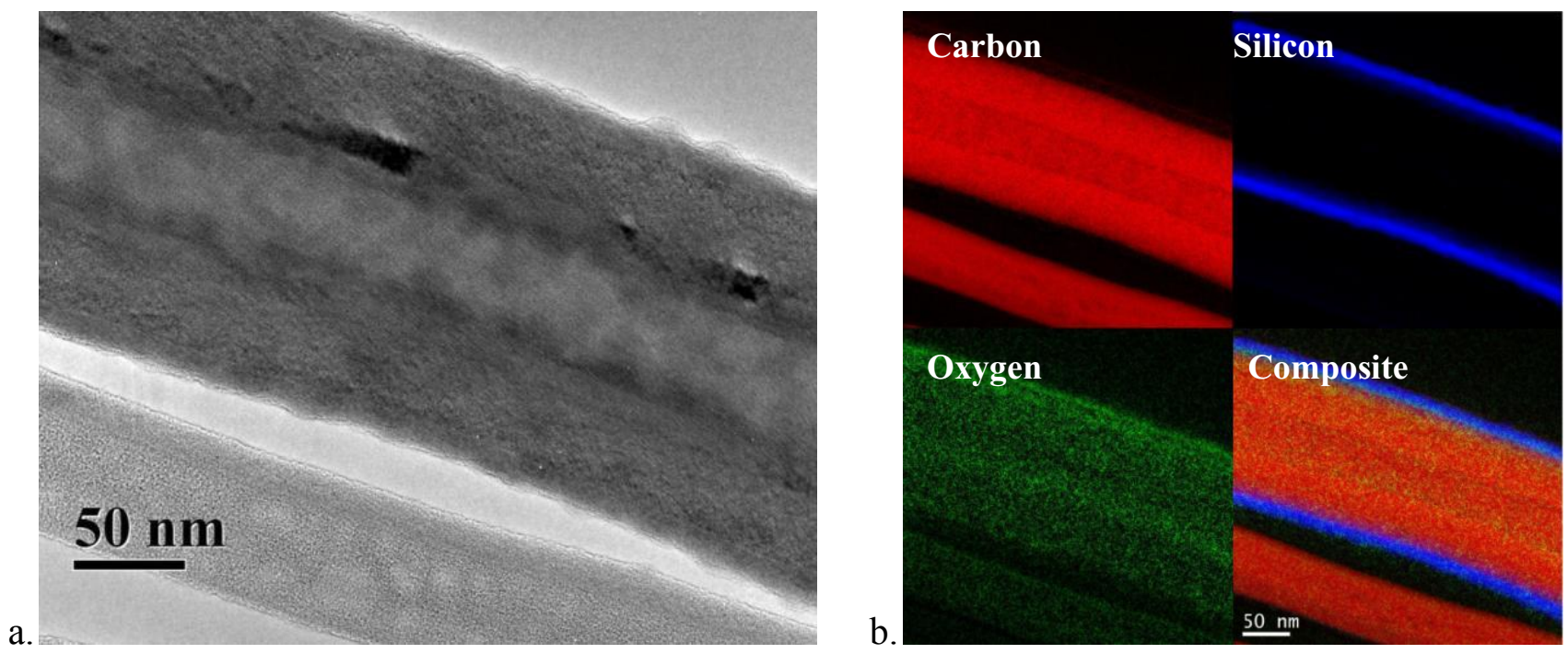

Figure 1. Transmission electron microscopy images of a fresh Si-CNF. (a. bright field image and b. energy filtered elemental maps $\mathrm{C}-\mathrm{K}, \mathrm{Si}-\mathrm{L}, \mathrm{O}-\mathrm{K}$ and composite maps)
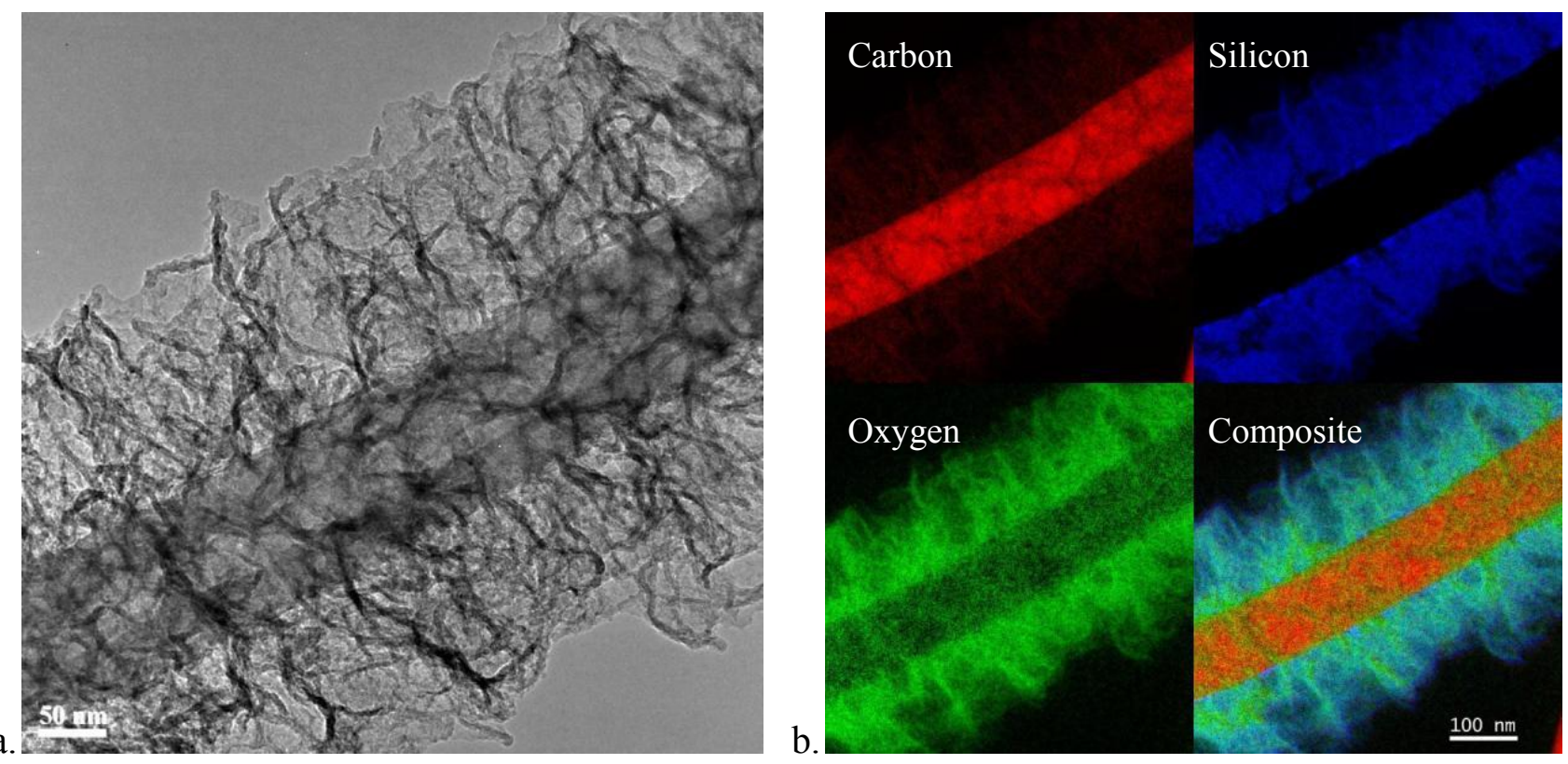

Figure 2. Transmission electron microscopy images of a cycled Si-CNF. (a. bright field image and b. energy filtered elemental maps $\mathrm{C}-\mathrm{K}, \mathrm{Si}-\mathrm{L}, \mathrm{O}-\mathrm{K}$ and composite maps) 